Preprint typeset in JINST style - HYPER VERSION

\title{
A concept for the experimental determination of the nucleon electric to magnetic form factor ratio at very Iow $Q^{2}$
}

\author{
G. Ron ${ }^{a *}$ E. Piasetzky ${ }^{b}$, and B. Wojtsekhowski ${ }^{c}$ \\ ${ }^{a}$ Department of Particle Physics, Weizmann Institute of Science, Rehovot 76100, Israel \\ (guy.roneweizmann.ac.il \\ ${ }^{b}$ The Beverly and Raymond Sackler School of Exact Sciences, Tel Aviv University, Tel Aviv 69978, \\ Israel \\ ${ }^{c}$ Thomas Jefferson National Accelerator Facility, Newport News, VA 23606, USA
}

\begin{abstract}
Stationary target measurements of the nucleon form factors have been performed with high precision down to $Q^{2}$ of $\sim 0.01 \mathrm{GeV}^{2}$ for protons $\left(G_{E}^{p}\right)$ and down to $\sim 0.1 \mathrm{GeV}^{2}$ for neutrons $\left(G_{M}^{n}\right)$. Conventional extraction using cross section and polarization measurement cannot be extended to very low values of $Q^{2}$ due to inherent experimental limitations. We present a proposal for a new approach to a measurement, using colliding beams, which will extend the range of possible measurement at low $Q^{2}$ by several orders of magnitude over stationary target limits.
\end{abstract}

\footnotetext{
${ }^{*}$ Corresponding author.
} 


\section{Introduction}

The Dirac and Pauli form factors of the nucleon, $F_{1}$ and $F_{2}$, commonly expressed as the Sachs electric and magnetic form factors [1]

$$
\begin{aligned}
G_{E} & =F_{1}-\tau F_{2} \\
G_{M} & =F_{1}+F_{2}
\end{aligned}
$$

where $\tau=Q^{2} / 4 M_{N}^{2}$ and $M_{N}$ is the nucleon mass, are fundumental observables describing the electromagnetic current of the nucleon,

$$
\left\langle p^{\prime}, \lambda^{\prime}\left|J^{\mu}(0)\right| p, \lambda\right\rangle=\bar{u}\left(p^{\prime}, \lambda^{\prime}\right)\left(\gamma^{\mu} F_{1}\left(Q^{2}\right)+i \frac{\sigma^{\mu \alpha}}{2 M} q_{\alpha} F_{2}\left(Q^{2}\right)\right) u(p, \lambda) .
$$

For a recent review of the nucleon form factors see [2] and references therein.

In the Breit frame the electric (magnetic) form factor is related to the charge (magnetization) density distribution by a Fourier transform,

$$
G_{E(M)}\left(Q^{2}\right)=\int \rho_{C h(M)}(\vec{r}) e^{-i \vec{q} \cdot \vec{r}} d^{3} r
$$

where $\vec{q}$ is the three momentum transfer and $Q^{2}=-q^{2}=\vec{q}^{2}-\omega^{2}$, and $\omega$ is the energy transfer $\left(\omega=E-E^{\prime}\right)$. Using this definition one may extract the Breit frame RMS charge and magnetization radii via a low $Q^{2}$ expansion of the form factors,

$$
\left\langle r^{2}\right\rangle=-\left.6 \frac{d G\left(Q^{2}\right)}{d Q^{2}}\right|_{Q^{2}=0},
$$

or, equivalently, by fitting the slope of the form factor at very low $Q^{2}$. While this extraction is strictly only valid in the non-relativistic regime and in the Breit frame it has been shown [3, 丹] that by using light-front formalism it is possible to relate the Dirac and Pauli form factors to the transverse Dirac and Pauli radii via a two dimensional Fourier transform,

$$
F_{1(2)}\left(Q^{2}\right)=\int \rho(\vec{b})_{D(P)} e^{-i \vec{q} \cdot \vec{b}} d^{2} b
$$

where $\vec{b}$ is the transverse radius vector. Note that eq. (1.4) is valid relativistically and in all reference frames. It was also shown [ $₫$ that it is possible to relate the difference of the RMS transverse Dirac and Pauli radii,

$$
\begin{aligned}
& F_{1}\left(Q^{2}\right) \approx 1-\frac{Q^{2}}{4}\left\langle b^{2}\right\rangle_{D}, \\
& F_{2}\left(Q^{2}\right) \approx \kappa\left(1-\frac{Q^{2}}{4}\left\langle b^{2}\right\rangle_{P}\right),
\end{aligned}
$$

where $\left\langle b^{2}\right\rangle$ is the RMS transverse radius, and the relation is valid for low $Q^{2}$. Eq. (1.5) was used to show that the magnetization density of the proton extends further than the charge density, however, the paucity of the data at low $Q^{2}$ is detrimental to an accurate determination of the difference. 
The low Q2 property of the nucleon is a subject of chiral QCD [5]. It is of great interest for to discover at what $Q^{2}$ predictions of chiral QCD are in agreement with the nature.

We further note that the values of the form factors at low $Q^{2}$ effect several other experimental results and calculations, such as, for example, calculations of the Zemach radius [6], the extraction of Generalize Parton Distributions (GPDs) [7] from experiment (via the Bethe-Heitler interference terms), and the extraction of strange form factors from parity violation measurements [8].

We conclude that accurate measurements of the nucleon form factors at low $Q^{2}$ are desirable, as evident in the approval of several recent experiments [9, 10] targeting the low $Q^{2}$ energy range.

\section{Form Factor Measurements}

The cross section for elastic scattering of an electron off the nucleon may be written as [11]:

$$
\begin{aligned}
\frac{d \sigma}{d \Omega_{e}} & =\left(\frac{d \sigma}{d \Omega}\right)_{M o t t} \frac{E^{\prime}}{E}\left\{F_{1}^{2}\left(Q^{2}\right)+2\left(F_{1}\left(Q^{2}\right)+F_{2}\left(Q^{2}\right)\right)^{2} \tan ^{2} \frac{\theta_{e}}{2}\right\} \\
& =\left(\frac{d \sigma}{d \Omega}\right)_{M o t t} \frac{1}{1+\tau}\left(G_{E}\left(Q^{2}\right)^{2}+\frac{\tau}{\varepsilon} G_{M}\left(Q^{2}\right)^{2}\right)
\end{aligned}
$$

where

$$
\left(\frac{d \sigma}{d \Omega}\right)_{M o t t}=\left(\frac{e^{2}}{2 E}\right)^{2}\left(\frac{\cos ^{2} \frac{\theta_{e}}{2}}{\sin ^{4} \frac{\theta_{e}}{2}}\right),
$$

is the Mott cross section for the scattering of a spin 1/2 electron from a spin-less, point-like target, $E / E^{\prime}=\left(1+2 E / M_{p} \sin ^{2} \theta_{e} / 2\right)^{-1}$ is the recoil factor, $\theta_{e}$ is the scattered electron angle, and $\varepsilon \equiv$ $\left[1+2(1+\tau) \tan ^{2}\left(\theta_{e} / 2\right)\right]^{-1}$ is the polarization of the virtual photon.

The traditional method of extracting the EM form factors is the so called "Rosenbluth separation" method [11] in which the EM cross section is measured for the same value of $Q^{2}$ and different values of $\varepsilon$ and a linear fit is used to determine the form factors. While this method has provided valuable data over the years, there are intrinsic limitations to the technique, easily discerned by examination of eq. (2.2). At high $Q^{2}$, the cross section is dominated by the term proportional to $G_{M}^{2}$, making an extraction of the $G_{E}$ term increasingly difficult. Conversely, at low $Q^{2}$ the $G_{M}$ term is suppressed by $Q^{2}$. Some experiments have measured the cross section at $\theta_{e} \sim 180^{\circ}$, where $\varepsilon=0$ and the cross section contains only the $G_{M}$ term, while useful, these measurements must take into account the finite detector acceptance which allows contamination of the measurement from the $G_{E}$ term.

In the last two decades an alternative method has been developed and employed. Using the high current, high polarization beams and highly polarized targets which have become available. In a recoil polarization measurement a longitudinally polarized electron beam is elastically scattered off an unpolarized nucleon and the polarization of the outgoing nucleon is measured. The polarization components of the outgoing nucleon (in the Born approximation) are [12]:

$$
\begin{aligned}
\sigma_{r e d} C_{x} & =-2 h \cot \frac{\theta_{e}}{2} \sqrt{\frac{\tau}{1+\tau}} G_{E} G_{M}, \\
\sigma_{r e d} C_{z} & =h \frac{E+E^{\prime}}{M_{N}} \sqrt{\frac{\tau}{1+\tau}} G_{M}^{2},
\end{aligned}
$$


where $C_{z}$ is the longitudinal polarization, $C_{x}$ is the transverse, in plane, polarization of the scattered nucleon, $\sigma_{\text {red }}$ is the reduced cross section,

$$
\sigma_{r e d}=(1+\tau) \frac{d \sigma / d \Omega}{(d \sigma / d \Omega)_{M o t t}}=G_{E}^{2}+\frac{\tau}{\varepsilon} G_{M}^{2},
$$

$h$ is the beam helicity, and all other quantities are defined in (2.1). Note that in the Born approximation the induced polarization, normal to the scattering plane, vanishes. Thus, the ratio of the electric to the magnetic form factors can be easily determined as:

$$
\mu_{N} \frac{G_{E}}{G_{M}}=-\mu_{N} \frac{C_{x}}{C_{z}} \frac{E+E^{\prime}}{2 M_{N}} \tan \frac{\theta_{e}}{2}
$$

Figure 1 presents an illustration of the recoil polarimetry method.

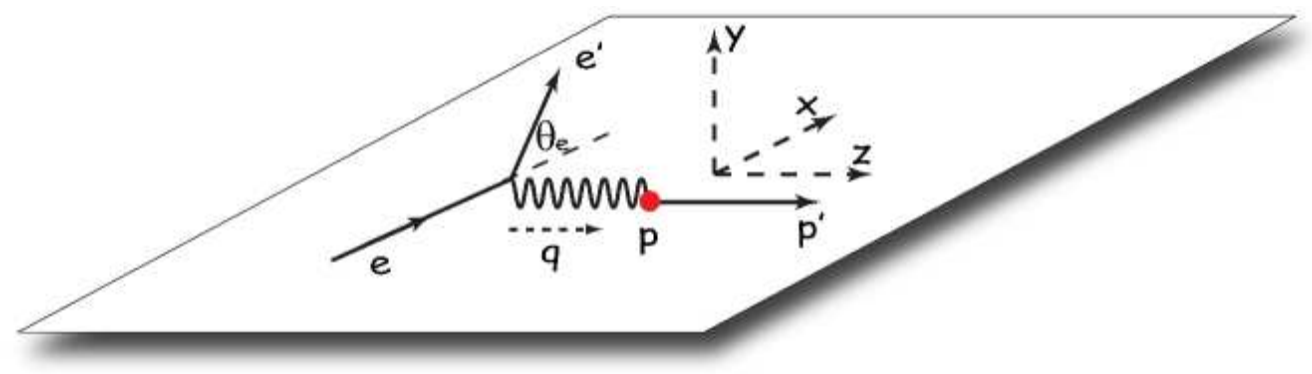

Figure 1. A schematic illustration of the recoil polarimetry method.

In a Beam Target Asymmetry measurement a longitudinally polarized electron beam is scattered off a polarized target and the asymmetry of the scattering cross section is measured. The asymmetry may be written as (neglecting the target dilution factor):

$$
\begin{aligned}
A & \equiv \frac{\sigma_{+}-\sigma_{-}}{\left(\sigma_{+}+\sigma_{-}\right)} \\
& =-\frac{2 \sqrt{\frac{\tau}{1+\tau}} \tan \frac{\theta}{2}\left\{\sqrt{\tau\left(1+(1+\tau) \tan ^{2} \frac{\theta}{2}\right)} \cos \theta^{*} G_{M}^{2}+\sin \theta^{*} \cos \phi^{*} G_{M} G_{E}\right\}}{\left(\frac{G_{e}^{2}+\tau G_{M}^{2}}{1+\tau}+2 \tau G_{M}^{2} \tan ^{2}(\theta / 2)\right)},
\end{aligned}
$$

where $\theta^{*}\left(\phi^{*}\right)$ is the polar (azimuthal) angle of the momentum vector of the recoil nucleon with respect to the target polarization vector. Figure 2 2 illustrates the beam target asymmetry method.

While spin correlation measurements have enabled the extension of the measured range of the form factor ratio to $Q^{2}$ as low as $0.01 \mathrm{GeV}^{2}$ [9] there exist fundamental difficulties in extending the range even further which may not be overcome in fixed-target or in-beam target experiments.

In a fixed target experiment the energy of the recoil nucleon in elastic scattering is directly proportional to the value of $Q^{2}$, namely,

$$
T_{N}=\omega=\frac{Q^{2}}{2 M_{N}}
$$




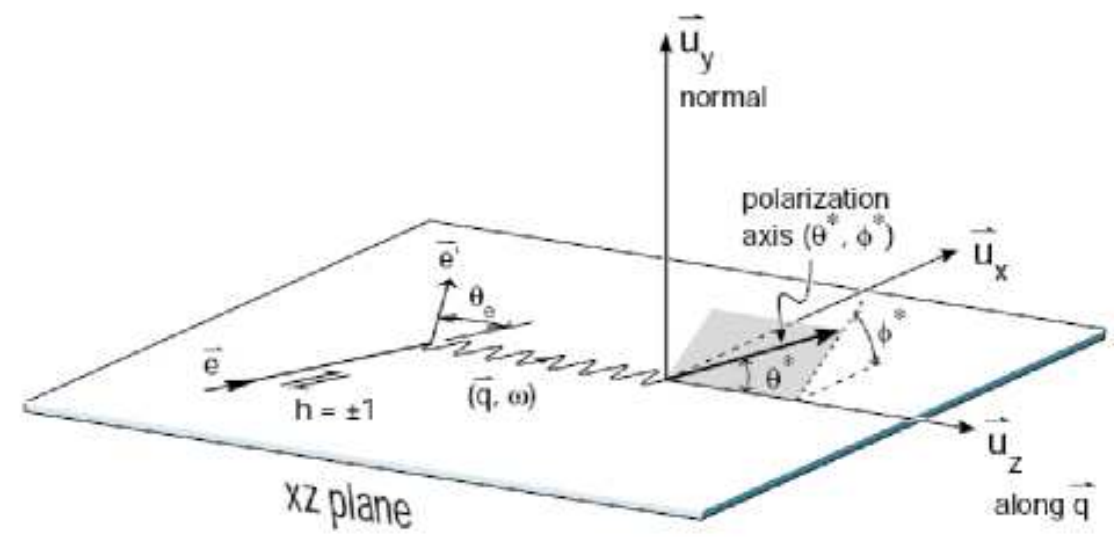

Figure 2. An illustration of the the reaction $\vec{p}\left(\vec{e}, e^{\prime}\right) p$.

where $T_{N}$ is the kinetic energy of the recoil nucleon. Thus, for low $Q^{2}$ the nucleon is ejected with very low kinetic energy, severely restricting the possibility of detecting it (even before requiring a secondary scattering reaction). One might suppose that by using beam-target asymmetry measurements it is possible to forgo detection of the recoil nucleon and detect instead the scattered electron. However, for low $Q^{2}$, the electron is ejected with very forward angle, again restricting the applicability of this method (cf. for example [9]).

\section{A new proposal}

Here we present a proposal for an alternative technique, based on the recoil polarization method, which will enable form factor ratio measurements to be extended to extremely low values of $Q^{2}$. The proposed measurement is based on the fact that $Q^{2}$ is a Lorentz invariant quantity. We propose to use two colliding beams, of unpolarized nucleons and of longitudinally polarized electrons, such that for low values of $Q^{2}$ the colliding particles retain almost all of their original momentum (in the lab frame), and are easy to detect. The polarization of the scattered nucleon may then be measured using a polarimeter, and the polarization of the nucleon in the rest frame may be calculated using a Lorentz transformation (which, as stated previously, leaves $Q^{2}$ unchanged). An alternative method, with the advent of polarized storage rings, is to collide two polarized beams and detect the scattered electron (analogous to the beam target asymmetry measurement).

An idea of the strength of this technique may be gained by examining some representative kinematic setting for a $500 \mathrm{MeV}$ electron beam and a $40 \mathrm{MeV}$ (kinetic energy) proton beam, which are summarized in Table 1 (where the initial electron beam angle is $0^{\circ}$ and that of the proton beam is $180^{\circ}$ ). Clearly, the scattered particles may be detected, even for very low value of $Q^{2}$.

An illustrative representation of the $Q^{2}$ range of the different experimental techniques is presented in Figure 3, the possible relative (statistical) uncertainties on the form factor ratio, $\Delta R / R$ are also shown. In order to compare the different methods we use the following assumptions:

- Scattered particles may be detected at angles of $5^{\circ}$ to $120^{\circ}$. 


\begin{tabular}{|c|c|c|c|c|}
\hline $\begin{array}{c}Q^{2} \\
\left(\mathrm{GeV}^{2}\right)\end{array}$ & $\begin{array}{c}\theta_{e} \\
(\mathrm{deg})\end{array}$ & $\begin{array}{c}E_{e}^{\prime} \\
(\mathrm{GeV})\end{array}$ & $\begin{array}{c}\theta_{p} \\
(\mathrm{deg})\end{array}$ & $\begin{array}{c}T_{p^{\prime}} \\
(\mathrm{GeV})\end{array}$ \\
\hline 0.55 & 111.55 & 0.403 & 134.75 & 0.138 \\
\hline 0.1 & 37.56 & 0.482 & 61.58 & 0.058 \\
\hline 0.01 & 11.5 & 0.498 & 20.53 & 0.042 \\
\hline 0.001 & 3.62 & 0.499 & 6.54 & 0.0402 \\
\hline 0.0006 & 2.8 & 0.499 & 5.066 & 0.0401 \\
\hline 0.0001 & 1.14 & 0.499 & 2.07 & 0.040 \\
\hline
\end{tabular}

Table 1. Representative kinematics for a $500 \mathrm{MeV}$ electron beam and a $40 \mathrm{MeV}$ proton beam.

- The beam energy varies between $200 \mathrm{MeV}$ and $4.4 \mathrm{GeV}$.

- Secondary scattering in a polarimeter is possible for protons whose kinetic energy exceed 40 $\mathrm{MeV}$.

- In order to remove the dependence on beam time allocation we assume that enough beam time is allocated to measure the cross section with $1 \%$ uncertainties and the polarizations with the same uncertainty (due to the large cross section at low $Q^{2}$ this is a valid assumption, cf. [9, 10]).

- For the colliding beams we assume a $500 \mathrm{MeV}$ electron beam colliding with a $40 \mathrm{MeV}$ proton beam.

- For clarity we omit regions of $Q^{2}$ where the statistical uncertainty is larger than $5 \%$.

Table 2 gives an overview of the restrictions on the different methods. For each method in Fig. 3 the high and low $Q^{2}$ cutoff values are explained.

\begin{tabular}{|l|c|c|}
\hline Method & Low $Q^{2}$ Cutoff & High $Q^{2}$ Cutoff \\
\hline Collider $\vec{e}\left(\vec{p}, e^{\prime}\right)$ & $\theta_{e^{\prime}}<5^{\circ}$ & $\theta_{e^{\prime}}>120^{\circ}$ \\
\hline Collider $\vec{e}\left(p, \overrightarrow{p^{\prime}}\right)$ & $\theta_{p^{\prime}}<5^{\circ}$ & $\theta_{p^{\prime}}>120^{\circ}$ \\
\hline Beam target asymmetry & $\theta_{e^{\prime}}<5^{\circ}$ & - \\
\hline Recoil polarization & $T_{p^{\prime}}<40 \mathrm{MeV}$ & - \\
\hline Rosenbluth separation & $\Delta R / R>5 \%$ & $\Delta R / R>5 \%$ \\
\hline
\end{tabular}

Table 2. Restricting parameters for the high and low $Q^{2}$ cutoff values of each method.

Figure 4 shows several $Q^{2}$ angle vs. electron beam energy and $T_{p}$ vs. beam energy contours for a proton beam of $40 \mathrm{MeV}$ energy colliding with an electron beam of energy between $100 \mathrm{MeV}$ and $1.5 \mathrm{GeV}$.

In order to present a quantitative idea about the applicability of this method we calculate the luminosity for a possible setup (electron storage ring and proton beam parameters are taken from the design specs for the ELISe ring [13] and the SARAF accelerator [14], respectively). Table 3 


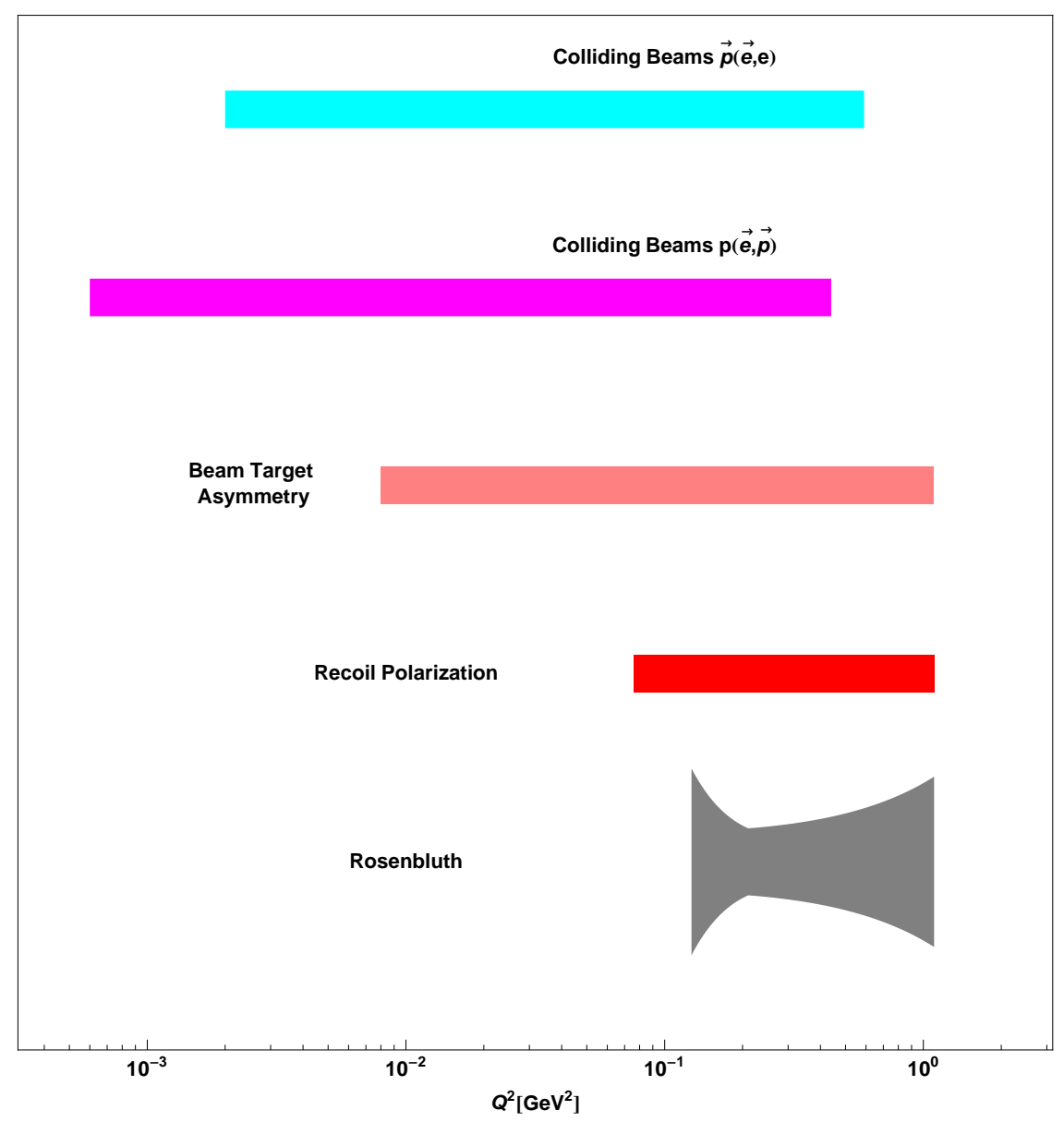

Figure 3. An illustration of the the ranges of applicability and the uncertainties for the different methods.

lists the parameters used for the electron storage ring and the linear proton beam. The calculated luminosity is $\mathscr{L} \sim 10^{29} \mathrm{~cm}^{-2} \mathrm{sec}^{-1}$. Using these values for $Q^{2}=0.0005 \mathrm{GeV}^{2}$ the calculated cross section is $d \sigma / d \Omega \sim 5.3 \cdot 10^{-26} \mathrm{~cm}^{-2}$ which translates into an event rate of $\mathscr{O}(10 \mathrm{~Hz})$ for a few mrad detector acceptance.

\section{Extensions}

Further intriguing extensions of this method are possible, here we note a few of the more interesting ones:

- A storage ring may be designed to accommodate both electron and positron beam and both proton and anti-proton beams are either available or will be in the near future. Thus, a comparison may be made of the measured asymmetries for the reactions:

$$
\begin{aligned}
& e^{-}+p \rightarrow e^{-}+p \\
& e^{+}+p \rightarrow e^{+}+p \\
& e^{-}+\bar{p} \rightarrow e^{-}+\bar{p} \\
& e^{+}+\bar{p} \rightarrow e^{+}+\bar{p}
\end{aligned}
$$



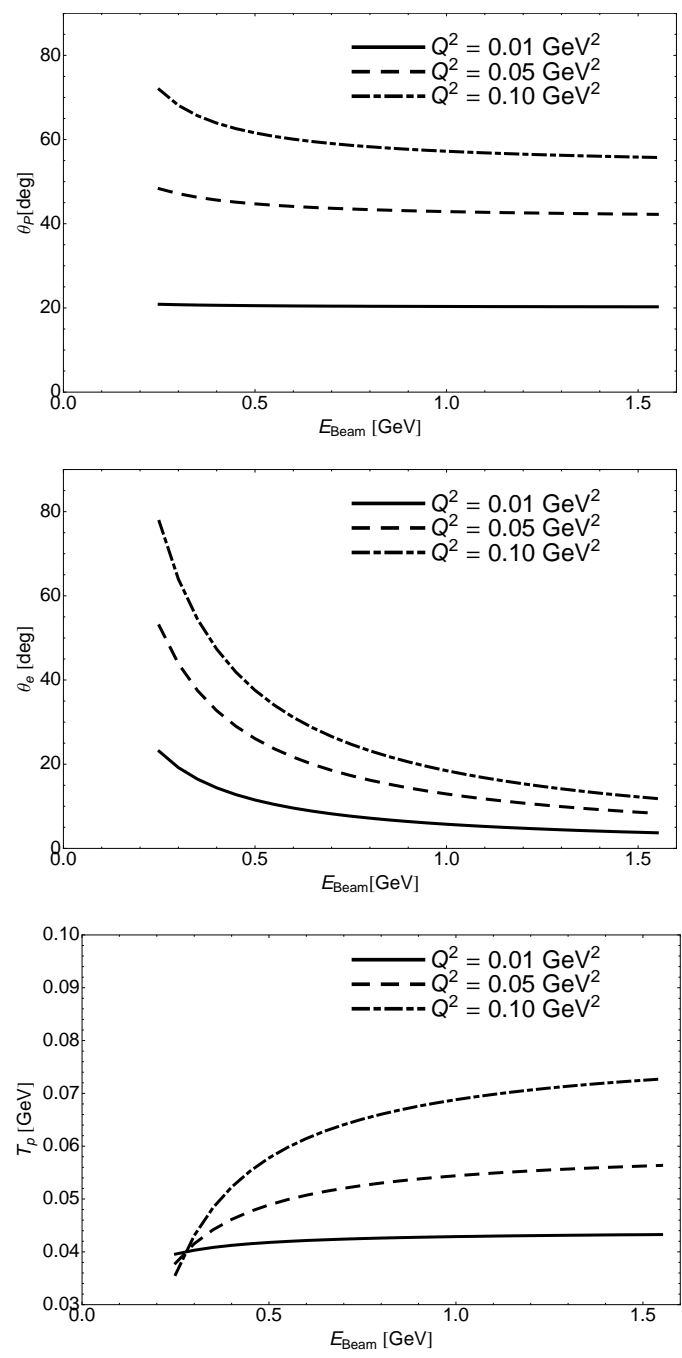

Figure 4. $\theta_{e}, \theta_{p}$ and $T_{p}$ vs. electron beam energy for several different $Q^{2}$ values.

which may be used as a test for the equivalence under $\mathrm{C}$ parity of the proton and anti-proton.

- Availability of a polarized proton beam (such as the one planned for the FAIR facility at GSI/Darmstadt[15]) will remove the need for the measurement of the outgoing proton polarization, requiring instead the (perhaps easier) measurement of the asymmetry in the $\vec{p}\left(\vec{e}, e^{\prime}\right)$ or $\vec{p}\left(\vec{e}, p^{\prime}\right)$ reaction.

\section{Detection Scheme}

In the simple 2-body reaction envisioned here detection of one of the final particles determines the kinematics of the reaction. The proposed approach for measurement of the form factors allows the proton to have significant energy after the scattering, even for very small momentum transfer, drastically simplifying its detection. 


\begin{tabular}{|ll|}
\hline Electron Storage Ring & \\
Circumference $(\mathrm{m})$ & 45.215 \\
Energy $(\mathrm{MeV})$ & 250 \\
Number of Bunches & 8 \\
Revolution Freq. (MHz) & 6.63 \\
Bunch Population & $5 \cdot 10^{10}$ \\
Current (mA) & 425 \\
Beam Emittance, $\varepsilon_{x, y}(\mu \mathrm{m} \cdot \mathrm{mrad})$ & 50 \\
Beam Size at IP, $\sigma_{x, z}(\mu \mathrm{m})$ & 220 \\
Bunch Length $(\mathrm{cm})$ & 4 \\
Total RF Generator Power $(\mathrm{kW})$ & 42 \\
\hline Proton beam & \\
Energy (MeV) & 40 \\
Current (mA) & 2 \\
Bunch Frequency $(\mathrm{MHz})$ & 176 \\
Bunch Population & $7 \cdot 10^{10}$ \\
Beam Radius at IP $(\mathrm{mm})$ & 0.6 \\
\hline
\end{tabular}

Table 3. Parameters used for the luminosity estimation [13, 14,.

When the proton beam is polarized, the final proton could be detected, for example, by a semiconductor detector with very good energy and position resolutions, leading to simple selection of the elastic scattering events.

Detection of scattered electrons with good energy resolution is possible by making use of the storage ring magnet to perform momentum analysis (for small scattering angles) or an ordinary magnetic spectrometer for larger angles.

For proton (antiproton) energies of several tens of $\mathrm{MeV}$ polarimetery via secondary scattering becomes an attractive option as the figure of merit for such polarimeters can be as high as $1 \%$ (see for example [16] and references therein). Since the luminosity of the proposed experiments will be on the level of $10^{30}-10^{32} \mathrm{~cm}^{-2} \mathrm{~s}^{-1}$ we expect no problems with the operation of segmented detectors even at small angles.

\section{Summary}

In summary, we have presented a method by which the nucleon electric to magnetic form factor ratio may be measured to high precision and low momentum transfer. Such a measurement will allow an accurate determination of the difference of the nucleon electric and magnetic radii, as well as impact many atomic high precision experiments. The experimental requirements to perform such a measurement are well understood and several possible facilities exist which may be able, with slight modifications, to perform such a measurement.

We thank R. Gilman for many illuminating discussions. We thank Y. Hammer for the kinematical calculations. We also thank the Israeli Science Foundation for partial support of this work. 
This work was partially supported by DOE contract DE-AC05-84ER40150 under which the Southeastern Universities Research Association (SURA) operated the Thomas Jefferson National Accelerator Facility.

\section{References}

[1] R. G. Sachs, High-Energy Behavior of Nucleon Electromagnetic Form Factors, Phys. Rev. 126, 2256 (1962).

[2] C. F. Perdrisat, V. Punjabi and M. Vanderhaeghen, Nucleon electromagnetic form factors, Prog. Part. Nucl. Phys. 59, 694 (2007), [hep-ph/0612014].

[3] G. A. Miller, Charge density of the neutron, Phys. Rev. Lett. 99, 112001 (2007), [0705.2409].

[4] G. A. Miller, E. Piasetzky and G. Ron, Proton electromagnetic form factor ratios at low $Q^{2}$, Phys. Rev. Lett. 101, 082002 (2008).

[5] V. Bernard and U. G. Meissner, Chiral perturbation theory, Ann. Rev. Nucl. Part. Sci. 57, 33 (2007) [arXiv:hep-ph/0611231].

[6] A. C. Zemach, Proton structure and the hyperfine shift in hydrogen, Phys. Rev. 104, 1771 (1956). A. V. Volotka, V. M. Shabaev, G. Plunien and G. Soff, Zemach and magnetic radius of the proton from the hyperfine splitting in hydrogen, Eur. Phys. J. D33, 23 (2005), [physics/0405118]. V. A. Yerokhin and V. M. Shabaev, One-loop self-energy correction to the $1 s$ and 2 s hyperfine splitting in H-like systems, Phys. Rev. A64, 012506 (2001), [hep-ph/0103037].

[7] A. V. Radyushkin, Asymmetric gluon distributions and hard diffractive electroproduction, Phys. Lett. B385, 333 (1996), [hep-ph/9605431].

M. Diehl, Generalized parton distributions, Phys. Rept. 388, 41 (2003), [hep-ph/0307382].

X. Ji, Generalized parton distributions, Ann. Rev. Nucl. Part. Sci. 54, 413 (2004).

M. Vanderhaeghen, Overview of nucleon structure studies, Nucl. Phys. A805, 210 (2008), [0710.5200].

X. Ji, Gauge-invariant decomposition of nucleon spin, Phys. Rev. Lett. 78, 610 (1997).

[8] K. A. Aniol et al., Parity-violating electroweak asymmetry in $\vec{e} p$ scattering, Phys. Rev. C69, 065501 (2004), [nucl-ex/0402004].

[9] J. R. Arrington, D. Donal, D. W. Higinbotham, R. Gilman, G. Ron and A. Sarty, Jefferson Lab experiment E08-007, unpublished (2007), http://hallaweb.jlab.org/experiment/E08-007/home2_files/LowQ2Prop.pdf.

[10] P. Achenbach et al., Experimental proposal, Mainz A1 Collaboration, unpublished.

[11] M. N. Rosenbluth, High Energy Elastic Scattering of Electrons on Protons, Phys. Rev. 79, 615 (1950).

[12] A. I. Akhiezer and M. P. Rekalo, Polarization effects in the scattering of leptons by hadrons, Sov. J. Part. Nucl. 4, 277 (1974).

[13] H. Simon et al., NUSTAR/ELISe - Technical Proposal [20051215], unpublished (2006), http://www.gsi.de/documents/DOC-2006-Mar-118-1.pdf.

[14] C. Piel, K. Dunkel, F. Kremer, M. Pekeler, P. vom Stein, D. Berkovits and I. Mardor, Phase 1 Commissioning Status of the $40 \mathrm{MeV}$ Proton/Deuteron Accelerator SARAF, In the Proceedings of 11 th European Particle Accelerator Conference (EPAC 08), Magazzini del Cotone, Genoa, Italy, 23-27 Jun 2008, pp THPP038. 
[15] FAIR - Facility for Antiproton and Ion Research, http://www.gsi.de/fair/ .

[16] J. Glister et al., Proton Polarimeter Calibration between 82 and $217 \mathrm{MeV}$, arXiv:0904.1493 [nucl-ex]. 